\title{
Um Modelo para Dinâmica Populacional em Meios Heterogêneos
}

V.O. NEVES ${ }^{1}$, Departamento de Física, Estatística e Matemática, Universidade Regional do Noroeste do Estado do Rio Grande do Sul - UNIJUI, 98700-000 Ijuí, RS, Brasil

L.A.D. RODRIGUES ${ }^{2}$, D.C. MISTRO ${ }^{3}$, Departamento de Matemática, Universidade Federal de Santa Maria - UFSM, 97105-900 Santa Maria, RS, Brasil.

\begin{abstract}
Resumo. Em plantações economicamente importantes, as plantas estão em geral distribuídas em um padrão com textura regular e periódica. Neste trabalho, estudamos a influência do espaçamento e da alternância das plantas na dispersão de insetos herbívoros. Supomos que os insetos se movimentam microscopicamente por meio de uma pesquisa aleatória local representada por uma difusão clássica em uma escala macroscópica.

O meio é periódico apresentando alternadamente uma região favorável e outra menos favorável com diferentes taxas de dispersão.

As simulações deste modelo produzem um efeito cinético que demonstra visualmente a evolução de uma população no seu aspecto macroscópico. Questões como a heterogeneidade espacial podem ser incorporadas ao modelo discreto e testadas. A existência e estabilidade de padrões estacionários, periódicos ou ondas de invasão no plano são também facilmente observadas.
\end{abstract}

\section{Introdução}

Os mecanismos que geram os padrões de invasão herbívora não são importantes somente para compreender uma variedade de fenômenos em dinâmica de populações, mas são também de grande interesse econômico [3]. Uma praga na agricultura é um inseto que atinge densidades suficientemente altas para causar danos sobre plantas economicamente importantes. O aparecimento de pragas depende da distribuição espacial das plantas bem como do comportamento do herbívoro na busca de alimento [4].

O movimento dos insetos dentro de uma plantação, denominado de movimento trivial, é influenciado de maneira decisiva pela textura da vegetação. Como todos

\footnotetext{
1 evhopner@brturbo.com.br; mestranda em Modelagem Matemática.

2luizdiaz@ccne.ufsm.br; orientador de mestrado.

${ }^{3}$ diomar@ccne.ufsm.br; colaboradora.
} 
os insetos se envolvem em um movimento trivial durante a procura de alimentos, um mecanismo para a compreensão dos padrões deste movimento poderia ser muito útil.

Características espaciais da plantação, tais como a aglomeração de plantas hospedeiras ou a alternância entre áreas cultivadas e não cultivadas podem modificar o comportamento do inseto na procura do seu alimento. Um mecanismo muito freqüente observado em insetos envolve uma mudança no padrão de procura imediatamente após o encontro do alimento. A redução do comprimento e na velocidade do movimento e o pronunciado aumento nas mudanças de direção são as técnicas mais comuns utilizadas pelos insetos para permanecer em regiões onde os recursos são mais favoráveis. Esta tendência de intensificar a procura na vizinhança das presas recentemente capturadas ou de permanecer em regiões de maior qualidade das plantas denomina-se prospecção em área restrita. É importante observar que são as condições locais, isto é, o que o inseto "experimenta" na posição em que se encontra, que determinam as mudanças no comportamento de prospecção.

\section{Formulação do Modelo}

O presente modelo foi desenvolvido com o objetivo de estudar a movimentação de insetos herbívoros em uma plantação extensa com textura periódica [5] e [6]. As plantas estão distribuídas em fileiras (sulcos) apresentando alternadamente faixas cultivadas e não cultivadas.

O movimento dos insetos será considerado aleatório na procura de alimentos. Para levar em conta a tendência dos indivíduos permanecerem por mais tempo em regiões favoráveis, consideramos a dispersão menor sobre as fileiras com plantas e maior nas regiões entre os sulcos.

Construímos um modelo matemático discreto de comportamento microscópico com o principal propósito de produzir uma descrição macroscópica do fenômeno. Além de permitir uma conexão entre as diferentes escalas envolvidas, os modelos para simulação podem orientar a pesquisa biológica identificando parâmetros importantes que os entomologistas podem medir por meio de experimentos controlados $[4]$.

Neste modelo inicial, em que estamos mais interessados no processo dinâmico espacial da população, a dinâmica vital pode ser desprezada. O processo de interação será importante durante o período de colonização, o qual deve vir após a invasão. Portanto, a plantação será considerada estacionária e o número total de insetos mantido constante durante as simulações nesta primeira etapa.

O estado do sistema é representado atribuindo valores para a densidade populacional em cada vértice de um reticulado plano. A densidade de herbívoros no ponto $(i, j)$ na etapa $t$ é denotada por $u_{i j}^{t}$.

A regra de atualização para a densidade de insetos em cada célula levará em conta a movimentação dos indivíduos na direção de seus quatro vizinhos mais próximos. Portanto, a cada iteração, uma fração $\mu$ de insetos abandona sua posição distribuindo-se equitativamente entre os quatro vizinhos mais próximos, enquanto 
a fração restante $(1-\mu)$ permanece na sua posição original.

A equação para a dispersão dos insetos é:

$$
u_{i j}^{t+1}=(1-\mu) u_{i j}^{t}+\mu \bar{u}_{i j}^{t}
$$

onde $\bar{u}_{i j}^{t}$ é a média da população de insetos dos quatro vizinhos mais próximos.

Diversos mecanismos são propostos na literatura especializada na tentativa de explicar a tendência dos insetos permanecerem por mais tempo em regiões favoráveis. Neste trabalho, propomos uma alternativa simples: vamos considerar a fração constante $\mu$ de indivíduos que abandonam sua posição menor sobre as fileiras com plantas e maior nos espaços vazios entre as fileiras.

Para garantir uma maior confiabilidade nas predições do modelo, consideramos algumas funções de estado que são calculadas a cada etapa de tempo: número de indivíduos, distância quadrática média, distribuição espacial da população e velocidade de dispersão.

Durante uma escala de tempo compatível com um processo de invasão de uma plantação é razoável não incluir as dinâmicas vitais. Portanto, nesta etapa o número de indivíduos permanece constante e não há reposição ou perdas na plantação.

Uma medida adequada da dispersão em processos de invasão iniciados pontualmente é a média ponderada do quadrado das distâncias, também chamada distância quadrática média e definida como:

$$
\sigma^{2}=\frac{\sum u_{i j}^{t}\left[\left(i-i_{0}\right)^{2}+\left(j-j_{0}\right)^{2}\right]}{\sum u_{i j}^{t}},
$$

onde $\left(i_{0}, j_{0}\right)$ representa a origem do processo de dispersão.

\section{Simulações}

As simulações foram realizadas em um reticulado de $80 \times 80$ com os indivíduos liberados em uma única posição no centro do domínio.

O número de iterações foi calculado para garantir que o processo de dispersão não sofresse influência das fronteiras, isto é, na escala de tempo considerada, os insetos não atingem as fronteiras.

Consideramos uma plantação distribuída periodicamente no espaço, alternando fileiras com plantas e faixas vazias. Este padrão procura descrever macroscopicamente uma plantação mecanizada regularmente espaçada e de grandes dimensões.

Verificamos que uma taxa de movimentação menor sobre as fileiras com plantas produz uma maior concentração de insetos nas plantas hospedeiras (Figura 1).

Calculamos um coeficiente médio $\bar{\mu}$, dado por:

$$
\bar{\mu}=\frac{\left(l_{1}+l_{2}\right) \mu_{1} \mu_{2}}{l_{1} \mu_{2}+l_{2} \mu_{1}} .
$$




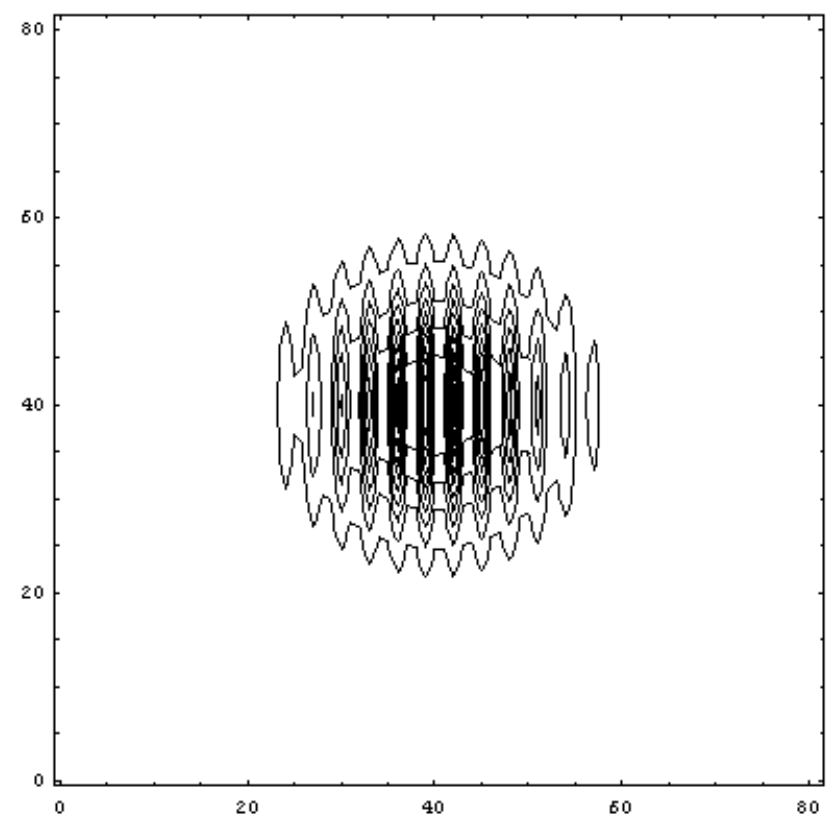

Figura 1: Distribuição espacial dos insetos após 200 iterações, em uma plantação por fileiras, para $\mu_{1}=0,4$ e $\mu_{2}=0,9$.

Observa-se uma maior concentração nas fileiras com plantas.

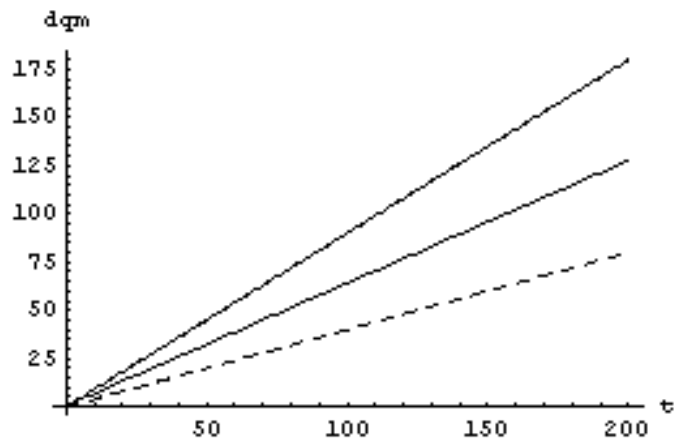

Figura 2: Distância quadrática média: $\mu=0,4$ (linha tracejada), $\mu=0,9$ (linha pontilhada) e com coeficiente periódico $\mu_{1}=0,4$ nas fileiras de plantas e $\mu_{2}=0,9$ nas faixas entre as fileiras (linha contínua). 
A Figura 2 apresenta uma comparação entre a distância quadrática média para difusão simples com coeficiente $\mu=0,4$ (linha tracejada) e $\mu=0,9$ (linha pontilhada) em um meio homogêneo e a distância quadrática média em um meio periódico com $\mu_{1}=0,4$ e $\mu_{2}=0,9$ (linha contínua).

O coeficiente médio dado em (3.1) confere com o coeficiente angular da reta central na Figura 2 que representa a distância quadrática média.

Observamos que, do ponto de vista macroscópico, a distância quadrática média no meio periódico é linear em t caracterizando um processo difusivo clássico com coeficiente de difusão "médio" $\bar{\mu}$.

\section{Conclusões e perspectivas}

Observamos, através das simulações, que uma redução no coeficiente de difusão dos insetos sobre as fileiras com plantas representa um mecanismo efetivo para explicar uma maior fixação dos insetos nas plantas.

Verificamos ainda que o processo de dispersão em um meio periódico (com coeficiente de difusão variando de maneira periódica) pode ser representado macroscópicamente por uma difusão clássica com coeficiente de difusão "médio". Este coeficiente de difusão médio é a média harmônica dos coeficientes de difusão do meio periódico $[2]$.

Uma extensão natural do modelo discreto é a obtenção de um modelo contínuo com um coeficiente efetivo médio $\bar{D}$. Este é um procedimento freqüente em fenômenos nos quais aparecem duas escalas espaciais: incorporar os efeitos da escala microscópica através de uma média apropriada tomada na escala macroscópica [1] e $[2]$.

Outro aspecto que deverá ser abordado futuramente é a incorporação da dinâmica vital da população. Para tal é necessário considerar, por exemplo, a taxa de crescimento periódica em relação à $x$, isto é, um maior crescimento em regiões favoráveis e um menor nas regiões menos favoráveis.

\footnotetext{
Abstract. In economically important plantations, the plants in general are distributed in a standard with regular and periodic texture. In this work we study the influence of the spacing and the alternation of the plants in the dispersion of herbivorous insects. We assume that the insects if put into motion microscopically in local random way represented by a classic diffusion in a macroscopic scale.

The environment is periodic alternatingly presenting a favorable region and another less favorable with different dispersion rate.

The simulations of this model produce a effect kinetic that visually demonstrate the evolution of a population in its macroscopic aspect. Questions as the space heterogeneity can be incorporated the discrete model and be tested. The existence and stability of stationary, periodic standards or waves of invasion in the plan also are easily observed.
} 


\section{Referências}

[1] M.H. Holmes, "Introduction to Perturbation Methods", Springer-Verlag, New York, 1995.

[2] J.B. Keller, Darcy's Law for Flow in Porous Media and the Two-Space Method, em "Nonlinear Problems in Mechanics", (R. Sternberg, ed.), pp. 429-443, Springer, 1980.

[3] J.D. Murray, "Mathematical Biology", Springer-Verlag, Berlin, 1993.

[4] L.A.D. Rodrigues, "O Acaso e a Decisão: Modelos Matemáticos para Dispersão Populacional", Tese de Doutorado, IMECC, UNICAMP, Campinas, SP, 1998.

[5] N. Shigesada e K. Kawasaki, "Biology Invasions: Theory and Practice", Oxford University Press, 1997.

[6] N. Shigesada, K. Kawasaki e E. Teramoto, Traveling periodic waves in heterogeneous environments, Theorical Population Biology, 30 (1986), 143-160. 